\title{
Orthodontic Attachment Adhesion to Ceramic Surfaces
}

\author{
Anca Labunet (D) \\ Andreea Kui ${ }^{2}$ \\ Andrada Voina-Toneal \\ Alexandra Vigu' \\ Sorina Sava' \\ 'Dental Materials Discipline, "Iuliu \\ Hatieganu" Medicine and Pharmacy \\ University Cluj-Napoca, Cluj, Romania; \\ ${ }^{2}$ Prosthodontics Discipline, "Iuliu \\ Hatieganu" Medicine and Pharmacy \\ University Cluj-Napoca, Cluj, Romania
}

This article was published in the following Dove Press journal: Clinical, Cosmetic and Investigational Dentistry

\begin{abstract}
Ceramic materials are constantly evolving, achieving good functionality and aesthetics. Bonding to ceramics may be difficult because of high toxicity procedures and risk of surface damage. The review aims to answer several research questions: Is there a golden standard for bonding to ceramic? Are there adhesives or types of photopolymerization lamps that produce a higher bond strength on certain types of ceramics rather than others? Articles focusing on the bonding process of orthodontic attachments to ceramic surfaces searched in Pubmed, Medline and Embase, published between 1990 and 2018 were revised. Exclusions concerned bonding to non-ceramic surfaces, bonding to ceramic surfaces that are not destined for orthodontics or laser usage. Forty-nine articles that matched the inclusion criteria were researched. The following categories of original research articles were compared and discussed: metallic brackets bonding to ceramic surfaces, ceramic brackets to ceramic surfaces, bonding to new types of ceramics, such as zirconia, lithium disilicate, different photopolymerisation devices used on bonding to ceramics. Some types of adhesive may achieve minimal bond strength (6-8 MPa) even on glazed ceramic. Ceramic surface preparation may be done by sandblasting or hydrofluoric acid $(60 \mathrm{~s}$ application and $9.6 \%$ ) with generally similar results. Studies rarely show any statistical difference and there are reduced number of samples in most studies. Ceramic brackets show better adhesion to ceramic surfaces and the same bonding protocol is advised. A higher bond strength may lead to ceramic surface. Few studies focus on newer types of ceramics; additional research is necessary. There is no clear evidence that a certain type of photopolymerization device produces higher shear bond strength values.
\end{abstract}

Keywords: ceramic bond, adhesion, shear bond strength

\section{Introduction}

As patient concerns for aesthetics are raising and ceramics is used for frontal and lateral areas in prosthodontics, orthodontists must choose from several modalities of bonding to ceramics. Ceramic materials are versatile and constantly evolving, making it possible to achieve good functionality and aesthetics. This review offers a thorough image of studies focusing on bonding to ceramic surfaces.

For a better understanding of the data provided by the researchers, it is important to understand the differences between several types of ceramics. We can define ceramics by the nature of their composition of glass-to-crystalline ratio. A simple classification based on microstructure categorizes ceramics into four basic categories:

- composition category 1 - glass-based systems (mainly silica),

- composition category 2 - glass-based systems (mainly silica) with fillers, usually crystalline (leucite or lithium disilicate), 
- composition category 3 - crystalline-based systems with glass fillers (mainly alumina)

- composition category 4 - polycrystalline solids (alumina and zirconia). ${ }^{1}$

Glass-based systems are made of materials that contain silicon dioxide (silica or quartz), which incorporates various amounts of alumina. Aluminosilicates found in nature are known as feldspars. Synthetic forms of aluminosilicate glasses are also manufactured for dental ceramics. ${ }^{1,2}$

Glass-based systems with fillers have a very large range of glass-crystalline ratios and crystal types. Thus, the category can be subdivided into three groups, among which the difference is that varying amounts of different types of crystals have either been added or grown in the glassy matrix. The primary crystal types are leucite, lithium disilicate or fluoroapatite. Subcategories include feldspathic porcelain, high leucite containing porcelain, lithium disilicate or IPS Empress II or IPS e.max. ${ }^{1,3,4}$

Crystalline-based systems with glass fillers are marketed under the name In-Ceram. The system was developed as an alternative to conventional metal ceramics. ${ }^{1}$

Polycrystalline solids are solid-sintered, monophase ceramics, that are formed by directly sintering crystals together without any intervening matrix to form a dense, air-free, glass-free, polycrystalline structure. They are either solid-sintered aluminous-oxide or zirconia-oxide frameworks. ${ }^{1,2}$

All materials can be processed by varied techniques, but a simple classification based on processing is powder/liquid, glass-based systems, machinable or pressable blocks of glass-based systems and $\mathrm{CAD} / \mathrm{CAM}$ or slurry, dieprocessed, mostly crystalline (alumina or zirconia) systems. ${ }^{5}$

Important research has been conducted on the adhesive bond between brackets and ceramics. Bracket type and base, adhesive type, ceramic type and prebonding procedures all influence the adhesive bond. Bonding to ceramics is harder to achieve because of hydrofluoric acid toxicity and low $\mathrm{pH}$, that can lead to the damage of the ceramic surface. Procedures such as air particle abrasion and other pre-treatments are needed to be performed prior to the actual definitive placement of the prosthetic restoration into the oral cavity.

Micro-etching, air abrasion or air particle abrasion is a technique in which particles of aluminum oxide, generally $50 \mu \mathrm{m}$, are projected in order to create abrasion on the surface of enamel or another substrate by high air pressure. ${ }^{6}$ When used for enamel surface preparation, air abrasion resulted in significantly lower shear bond strength, irrespective of the abrasive particle size, compared to that of using traditional acid etch technique. Air abrasion without acid etching resulted in significantly lower bond strength and it is not advised for clinical use. ${ }^{7}$

The shear bond strength or SBS is most often tested when studying adhesion, as it incorporates forces similar to masticatory ones and is conveniently produced in vitro. Good orthodontic bond strength is considered to be 5-10 $\mathrm{MPa}$ and should not exceed $40-50 \mathrm{MPa}$ as such a great force may damage enamel. ${ }^{8}$ This criteria may also be applied to ceramics, as damage to the vestibular surface of a veneer or crown may be considered after debonding.

There are also differences when bonding ceramic brackets instead of metallic brackets. The mean SBS of polycrystalline ceramic brackets was found to be significantly higher than that of stainless steel brackets in several studies when bonding to enamel. ${ }^{9-12}$

This review tries to answer several important questions the orthodontist needs to take into regard. Is there a golden standard for bonding to ceramic? Are there adhesives that produce a higher bond strength on certain types of ceramics rather than others? Are general rules of bonding that have proven correct for enamel surfaces also applicable to ceramics? Are there other factors that are seldom overlooked when choosing adhesive type and ceramic surface conditioning?

\section{Materials and Methods}

Databases Pubmed, Medline, Embase have been investigated by two independent researchers in February 2018, search terms: ceramics bond orthodontics, ceramics adhesion orthodontics. Exclusions have been made by reading titles and abstracts. The inclusion criteria were as follows: (1) articles focusing on the bonding process of orthodontic attachments to ceramic surfaces published between 1990 and 2018 were revised; (2) original researches; (3) experimental, in vitro studies. Exclusions criteria were as follows: (1) studies about bonding to non-ceramic surfaces; (2) bonding to ceramic surfaces that are not destined for orthodontics or laser usage for bracket base preparation or for ceramic surfaces (it involves technology that is not currently used by a majority of orthodontists). In February 2021, an update has been performed prior to publication of the article, following the same inclusion/ exclusion criteria, which led to 12 more articles included in the review. 
Researchers finally listed articles that focused on bonding metallic or ceramic brackets to ceramic surfaces by using regular means, available in most dental offices.

\section{Results}

Upon initial investigation, 98 articles were found. After carefully applying the exclusion criteria, 49 articles have been studied further. Upon update, 12 articles were added, which contain information published up to 2020. In order to evaluate the articles, we decided to divide them into three categories, based on the assessment technique: (a) studies assessing the bonding between metallic brackets and feldspathic ceramic materials; (b) studies assessing the bonding between esthetic brackets and feldspathic ceramic materials; (c) studies assessing the different types of brackets and different types of ceramic materials. Upon updating, 12 articles were added, mainly on the topic of bonding to non-feldspathic ceramics. They are all resumed in Tables 1-3.

\section{Discussion}

The aim of this study was to evaluate the literature regarding the bonding between different orthodontic brackets and ceramic materials. We selected 61 articles in this study. Exclusions have been made by reading titles and abstracts. Only experimental in vitro studies have been included, as clinical studies are difficult to reproduce and there are some uncontrollable factors, such as different use of appliances, doctor's capabilities.

A meta-analysis published in 2010 by Finnema and his team shows that many times, research articles do not take into consideration all factors that influence adhesion, such as storage of samples in water, photopolymerization time and crosshead speed. ${ }^{13}$ Reliability of studies increases when efforts are made to achieve similarity with the oral environment, for example, through thermocycling of the tested samples, as this influences bond strength. ${ }^{14-22}$ Throughout the articles included in this review, minimal bonding strengths were 6-8 $\mathrm{MPa}^{23}$

Many studies rely on mean shear bond strength when comparing different primers or different ceramic surface preparation techniques, as few of them obtain statistically significant results. There are few studies comparing certain types of adhesives, for example, Panavia or Concise, or primers, making it difficult to establish a reliable conclusion of the literature on a certain adhesive.
For an optimal evaluation process, all the studies included were divided according to the type of bracket and/or type of ceramic used in the research.

\section{Feldspathic Ceramic Surface and Metallic Brackets}

The first studies performed on feldspathic ceramic established that deglazed surfaces are better at achieving minimal adhesion for orthodontic forces. They used either sandpaper, acidulated phosphate fluoride, a coarse diamond bur or a Busch silent wheel to remove glazing. ${ }^{24,25}$ There are two studies, however, that contradict this finding, with similar results on glazed or deglazed ceramics. ${ }^{26,27}$

Silane use is generally considered to increase bond strength. In a study published in 1996, Zachrisson established that silane greatly increases tensile bond strength, ${ }^{28}$ results that coincided with Cochrane's. The latter also advised that the use of silane increased the occurrence of porcelain fracture at debonding. ${ }^{29}$ Another study supporting this is led by Chung in 1999, concluding that silane use means significantly higher shear bond strength, but it may also cause damage of the ceramic surface upon debonding. ${ }^{30}$ Bishara and collaborators find that the most reliable bonding procedure to ceramic surfaces is by using hydrofluoric acid and a silane before bonding, but this also produces the greatest damage to the porcelain surface. ${ }^{31}$ When comparing hydrofluoric acid concentrations of $4 \%$ or $9.6 \%$, Trakyali finds no statistically significant difference. ${ }^{32}$ In a study performed in 2019 , higher concentrations of hydrofluoric acid seem to increase SBS. ${ }^{20}$ Larmour et $\mathrm{al}^{33}$ by comparing phosphoric acid to hydrofluoric show that HF produces better results, confirmed also by other research teams. ${ }^{16,34-36}$ Contradicting this, Ajlouni and collaborators ${ }^{37}$ find two similar bonding sequences with similar results: HF, followed by silane and composite and phosphoric acid, followed by selfetching primer-adhesive and composite.

In 2003, a study conducted by Schmage contradicts this by obtaining the highest SBS through sandblasting and silane use or hydrofluoric acid without silane. Diamond roughening and sandblasting showed the highest surface roughness, but they can damage the ceramic surface. The results of this study are reliable, as thermocycling was done in 5000 cycles. ${ }^{38}$ A study published in 1999 by Bourke and his team reach a similar conclusion, that hydrofluoric acid or deglazing is unnecessary to achieve bond strength when using Right On bonding 
Table I Metallic Brackets Bonded to Feldspathic Ceramic Surfaces in Publishing Order

\begin{tabular}{|c|c|c|c|}
\hline $\begin{array}{l}\text { No. and } \\
\text { Year of } \\
\text { Publication }\end{array}$ & Adhesive Type & $\begin{array}{l}\text { Additional } \\
\text { Testing }\end{array}$ & Results \\
\hline $1993^{24}$ & Glazed/deglazed, different composites & & Inadequate SBS obtained, better when deglazed \\
\hline $1995^{25}$ & $\begin{array}{l}\text { Deglazed with sandpaper/deglazed with diamond bur/ } \\
\text { glazed, Acidulated phosphate fluoride on glazed/deglazed }\end{array}$ & & $\begin{array}{l}\text { APF - not enough SBS, mechanical retention needed, } \\
\text { improved by silane }\end{array}$ \\
\hline $1996^{28}$ & $\begin{array}{l}\text { Sandblasting/silane/HF9.6\%/4\% APF gels, either } \\
\text { Concise or 4-Meta resin }\end{array}$ & Thermocycling & $\begin{array}{l}\text { Silane sgn increases SBS, HF > APF gel, 4-Meta resin, } \\
\text { similar to Concise }\end{array}$ \\
\hline $1997^{29}$ & $\begin{array}{l}\text { Sandblasting/sandblasting+silane/HF/HF+silane/ } \\
\text { diamond bur + silane }\end{array}$ & & Highest SBS when silane was used, but risk of fracture \\
\hline $1998^{35}$ & $\begin{array}{l}\text { Diamond bur/HF } 8 \% / \text { microetching Al particles, } \\
\text { followed by silane+RightOn/silane+Concise/High- } \\
\text { Q-Bond without silane }\end{array}$ & SEM & $\begin{array}{l}\text { Silane+RightOn or silane+Concise }>\text { High-Q-Bond } \\
\text { without silane } \\
\text { HF>diamond bur, microetching }\end{array}$ \\
\hline $1999^{14}$ & $\begin{array}{l}\text { Deglazing/HF/phosphoric acid/silane, Right On } \\
\text { composite for all }\end{array}$ & Thermocycling & $\begin{array}{l}\text { HF produced damage, thermocycling sgn reduced SBS, } \\
\text { HF or deglazing unnecessary to achieve bond strength }\end{array}$ \\
\hline $1999^{30}$ & $\begin{array}{l}\text { Concise composite/Concise+ silane/Geristore/ } \\
\text { Geristore +silane/Fuji Ortho LC/Fuji+silane, HF } 9.6 \% \\
\text { for all }\end{array}$ & & $\begin{array}{l}\text { Silane groups sgn higher SBS, no sgn difference } \\
\text { between groups, ceramic damage in silanized groups } \\
\text { and Geristore }\end{array}$ \\
\hline $2002^{39}$ & $\begin{array}{l}\text { Control/diamond bur/HF } 10 \% / \text { sandblasted + } \\
\text { Scotchprime silane+ Concise }\end{array}$ & & All groups - adequate SBS \\
\hline $2003^{38}$ & $\begin{array}{l}\text { Diamond bur/sandblasting/HF 5\%/silica coating, self- } \\
\text { curing composite for all, with or without silane }\end{array}$ & Thermocycling & Highest SBS sandblasting +silane/HF without silane \\
\hline $2003^{23}$ & $\begin{array}{l}\text { Fuji LC/American Ortho Spectrum/Transbond/TP } \\
\text { orthodontics Python/Herculite, silane for all, } \\
\text { phosphoric acid }\end{array}$ & & Herculite and |TP orthodontics lower than 6-8 range \\
\hline $2005^{37}$ & $\begin{array}{l}37 \% \text { phosphoric acid +sealant+ adhesive }(\mathrm{I}) / \mathrm{HF}+\text { silane } \\
+ \text { composite }(2) / \text { phosphoric acid+self-etching }(3) \\
\text { primer-adhesive+composite }\end{array}$ & & Group 2 and 3 high, similar SBS, group I lowest \\
\hline $2005^{33}$ & $\begin{array}{l}\text { Phosphoric acid + Transbond/HF + Transbond/Fuji } \\
\text { Ortho LC + Transbond/Fuji Ortho LC +Phosphoric } \\
\text { acid, silane for all }\end{array}$ & & $\begin{array}{l}\text { Fuji had sgn lower SBS, phosphoric and HF not sgn } \\
\text { different }\end{array}$ \\
\hline $2005^{31}$ & $\begin{array}{l}37 \% \text { phosphoric acid }+ \text { Transbond/microetchingHF } \\
+ \text { silane+ Transbond/acid etch primer+ Transbond } / 35 \% \\
\text { phosphoric acid+cyanoacrylate adhesive }\end{array}$ & & $\begin{array}{l}\text { Cyanoacrylate lowest } \mathrm{SBS}<\text { conventional bonding }<\text { acid- } \\
\text { etch primer=HF group }\end{array}$ \\
\hline $2006^{26}$ & $\begin{array}{l}\text { Deglazed porcelain + Transbond XT/Glazed with } \\
\text { Transbond XT/deglazed +Fuji Ortho LC/Fuji Ortho LC }\end{array}$ & Thermocycling & No sgn difference between groups \\
\hline $2009^{32}$ & $\begin{array}{l}\text { HF } 9.6 \% / H F 5 \% \text {, Pulpdent Silane/Reliance silane, Unite } \\
\text { adhesive for all }\end{array}$ & & $\begin{array}{l}\text { Reliance sgn higher SBS than Pulpdent, concentration } \\
\text { of etching gels no sgn difference }\end{array}$ \\
\hline $2011^{75}$ & $\begin{array}{l}10 \% \text { HF 20s/60s, silane, Transbond XT, } 4 \text { different light } \\
\text { sources halogen, led, argon laser or plasma arc }\end{array}$ & & $\begin{array}{l}\text { No difference between light sources, } 20 \text { s etching, sgn } \\
\text { lower SBS }\end{array}$ \\
\hline $20 \mathrm{II}^{44}$ & $\begin{array}{l}\mathrm{HF}+\text { Embrace First Coat/HF +silane/sandblasting } \\
\text { +silane, Transbond XT for all, SSBS or cyclic shear } \\
\text { bond strength }\end{array}$ & & $\begin{array}{l}\text { SBS sgn higher than CSBS, sandblasting better than HF } \\
\text { groups, sandblasting+Embrace best results }\end{array}$ \\
\hline
\end{tabular}

(Continued) 
Table I (Continued).

\begin{tabular}{|c|c|c|c|}
\hline $\begin{array}{l}\text { No. and } \\
\text { Year of } \\
\text { Publication }\end{array}$ & Adhesive Type & $\begin{array}{l}\text { Additional } \\
\text { Testing }\end{array}$ & Results \\
\hline $2012^{41}$ & $\begin{array}{l}\text { HF } 10 \% 20 \mathrm{~s} / 60 \mathrm{~s} \text {, half with silane in each group, } \\
\text { Transbond XT/Fuji Ortho LC }\end{array}$ & & $\begin{array}{l}\text { Transbond XT higher SBS than Fuji, longer etching, } \\
\text { silane increases SBS }\end{array}$ \\
\hline $2012^{43}$ & $\begin{array}{l}\text { I0-MDP adhesive = Panavia } 2 / \text { TransbondXT/Fuji } \\
\text { Ortho LC }\end{array}$ & Thermocycling & Panavia highest mean SBS \\
\hline $2015^{32}$ & $\begin{array}{l}\text { I0\% HF. Transbond XT or Fuji Ortho LC, stored for } 24 \\
\text { h or } 6 \text { months in distilled water at } 37 \text { degrees C }\end{array}$ & & $\begin{array}{l}\text { Transbond XT SBS higher, storage time also influences } \\
\text { SBS }\end{array}$ \\
\hline $2015^{11}$ & $\begin{array}{l}\text { I0\%HF, Transbond XT for all, different light sources: } \\
\text { Radii Plus Led, XL } 2500 \text { halogen }\end{array}$ & $\begin{array}{l} \pm \\
\text { thermocycling }\end{array}$ & $\begin{array}{l}\text { Thermocycling and light sources influence bond } \\
\text { strength }\end{array}$ \\
\hline $2015^{13}$ & $\begin{array}{l}37 \% \text { phosphoric acid + silane/37\% phosphoric not } \\
\text { rinsed +silane/I0\% HF/I0\% HF+silane, Transbond for all }\end{array}$ & & $\mathrm{HF}$ and silane best SBS, but risk of ceramic fracture \\
\hline $2016^{16}$ & $\begin{array}{l} \pm 10 \% \text { HF, Clearfil Ceramic Primer CCP/ } \\
\text { RelyXCeramic Primer RCP, then Transbond XT }\end{array}$ & $\begin{array}{l} \pm \\
\text { thermocycling }\end{array}$ & $\begin{array}{l}\text { HF higher SBS, thermocycling reduced SBS, CCP silane } \\
\text { best amongst tested spec }\end{array}$ \\
\hline $2019^{20}$ & $\begin{array}{l}\text { HF: I-I\%;2-2.5\%;3-5\%;4-7.5\%;5-10\% (storage } 24 \text { h); } \\
6-1 \% ; 7-2.5 \% ; 8-5 \% ; 9-7.5 \% ; 10-10 \% \text { (thermal cycling) } \\
\text { for } 60 \text { s each + silane }\end{array}$ & $\begin{array}{l} \pm \\
\text { thermocycling }\end{array}$ & $\begin{array}{l}\text { The different HF acid concentrations } 5.0 \%, 7.5 \% \text { and } \\
10 \% \text { influenced the SBS. Thermocycling decreased SBS } \\
\text { significantly. }\end{array}$ \\
\hline $2019^{79}$ & $\begin{array}{l}10 \% \mathrm{HF} 60 \mathrm{~s}+2 \text { layers of silane, VALO Ortho } \\
\text { Cordless } 3200 \mathrm{~mW} / \mathrm{cm} 2 \text { for } 3 \mathrm{~s} / \text { Optilight Max } 1200 \\
\mathrm{~mW} / \mathrm{cm} 240 \mathrm{~s}\end{array}$ & $\begin{array}{l} \pm \\
\text { thermocycling }\end{array}$ & $\begin{array}{l}\text { No significant difference was observed between SBS } \\
\text { means in the different light activation devices used. The } \\
\text { samples subjected to thermocycling - lower SBS values. }\end{array}$ \\
\hline
\end{tabular}

system. ${ }^{14}$ In a study published by Sant'Anna, adequate SBS is achieved through either sandblasting, 10\% hydrofluoric acid or diamond bur roughening or no preparation at all when using Scotchbond silane and Concise. ${ }^{39}$

In conclusion, some adhesive types may achieve minimal bond strength even on glazed ceramic. Sandblasting may be a viable option for ceramic surface preparation, but it may be more difficult to use than HF. Statistical difference is rarely obtained, as there are few samples in each group for most studies. When using hydrofluoric acid, $60 \mathrm{~s}$ application and $9.6 \%$ solution are advised.

\section{Different Bonding Materials}

Many studies compare different adhesives. Composites have generally shown better SBS values than glass ionomers and newer types of silane are reliable options.

The Transbond XT bonding system, a golden standard for bonding to enamel surfaces is also tested on ceramic, showing more reliable results than glass-ionomer Fuji. ${ }^{26,40}$ In a study led by Pannes, ${ }^{23}$ by comparing Fuji, American Ortho Spectrum, Transbond, TP Orthodontics Python and Herculite bonding agents, only the TP system and
Herculite did not achieve the adequate bonding strength. Surface preparation was done with phosphoric acid and silane for all groups. Costa concludes that Transbond XT exhibits higher shear bond strengths than Fuji and longer etching and silane use increases shear bond strength. ${ }^{41} \mathrm{By}$ using 10\% hydrofluoric acid and either Transbond XT or Fuji Ortho LC, stored for $24 \mathrm{~h}$ or 6 months in distilled water at 37 degrees $\mathrm{C}$, Costa and his team of researchers found that the composite shows higher strength and storage time influences adhesion. ${ }^{42}$

Two studies contradict Transbond superiority. Sundareswaran found the best shear bond strength for Panavia, when comparing it to Transbond XT and Fuji Ortho LC. ${ }^{43}$ Lifshitz finds no significant difference between deglazed or glazed ceramic surface followed by either Transbond XT system or Fuji Ortho LC. ${ }^{26}$

Abdelnaby and his team research several aspects, starting with different primers: Embrace First-Coat primer and a regular silane have comparable shear bond strength. No significant difference was found when using either hydrofluoric acid and Embrace First-Coat or sandblasting and silane. ${ }^{44}$ Clearfil Ceramic Primer shows better results than 
Table 2 Esthetic Brackets Bonded to Feldspathic Ceramic Surfaces in Publishing Order

\begin{tabular}{|c|c|c|c|c|}
\hline $\begin{array}{l}\text { No. and } \\
\text { Year of } \\
\text { Publication }\end{array}$ & $\begin{array}{l}\text { Bracket } \\
\text { Type }\end{array}$ & Adhesive Type & $\begin{array}{l}\text { Additional } \\
\text { Testing }\end{array}$ & Results \\
\hline $2001^{48}$ & Ceramic & $\begin{array}{l}\text { Sandblasting } 50 \mathrm{um} / \mathrm{silane} / \text { sandblasting+silane/HF } 9.6 \% / \\
\text { sandblasting + 4-Meta adhesive, no-mix bonding } \\
\text { composite }\end{array}$ & & $\begin{array}{l}\text { HF+ silane sgn higher SBS, similar } \\
\text { tosandblasting+silane }\end{array}$ \\
\hline $2003^{46}$ & Ceramic & $\begin{array}{l}\mathrm{HF} / \text { microetching + Ideal I (without silane)/Right On } \\
\text { (with silane) }\end{array}$ & & $\begin{array}{l}\text { HF sgn higher SBS, both adhesives } \\
\text { satisfactory results }\end{array}$ \\
\hline $2003^{17}$ & Composite & $\begin{array}{l}\text { Self-cure non mixed resin Unite with either etching/ } \\
\text { silane/etch+silane }\end{array}$ & $\begin{array}{l}\text { Thermocycling } \\
\text { half of specs }\end{array}$ & $\begin{array}{l}\text { Highest SBS etch + silane, no } \\
\text { thermocycling }\end{array}$ \\
\hline $2004^{49}$ & Polycarbonate & $\begin{array}{l}\text { Phosphoric acid+primer+bonding agent/HF+primer } \\
\text { +bonding agent/tribochemical silica coating+silane/APA } \\
30 \text { um+silane+bonding agent, composite for all }\end{array}$ & Thermocycling & $\begin{array}{l}\text { Silica coating + silanizationsgn } \\
\text { higher SBS than phosphoric acid, } \\
\text { APA=HF groups }\end{array}$ \\
\hline $2006^{77}$ & Ceramic & $\begin{array}{l}\text { HF } 9.6 \% 2 \mathrm{~min}+\text { silane (I)/APA+HF } 9.6 \%+\text { silane (2)/APA } \\
+ \text { silane (3), Light Bond adhesive for all }\end{array}$ & Thermocycling & Group I=2, lower SBS in Group 3 \\
\hline $2006^{78}$ & Ceramic & $\begin{array}{l}\text { HF } 9.6 \% 2 \text { min+silane+adhesive, curing soft start LED or } \\
\text { halogen light }\end{array}$ & & Sgn higher SBS for LED lamp \\
\hline $2007^{76}$ & Ceramic & $\begin{array}{l}\text { APA } 25 \text { um+HF } 9.6 \%+\text { silane+ halogen curing unit for } 10 \mathrm{~s} \\
\text { or } 20 \text { s/LED } 3.5 \text { or } 10 \text { s in standard or fast mode }\end{array}$ & & $\begin{array}{l}\text { No sgn differences between SBS, } \\
\text { ARI scores, porcelain damage and } \\
\text { bracket base fracture. }\end{array}$ \\
\hline $2012^{52}$ & Ceramic & $\begin{array}{l}\text { Control/diamond bur +orthophosphoric } 37 \% / \mathrm{HF} 10 \% / \\
\text { HF 10\%+silane, Transbond XT for all }\end{array}$ & & $\begin{array}{l}\mathrm{HF}+\text { silane best SBS, but risk of } \\
\text { damage }\end{array}$ \\
\hline $2012^{50}$ & $\begin{array}{l}\text { Metallic and } \\
\text { ceramic }\end{array}$ & $\begin{array}{l}\text { HF } 9 \%+\text { silane for all, } 2 \text { composites, } 4 \text { light curing units, } \\
3 \text { led, I halogen }\end{array}$ & & $\begin{array}{l}\text { SBS sufficient in all groups, mean } \\
\text { SBS higher for ceramic brackets }\end{array}$ \\
\hline
\end{tabular}

RelyXCeramic Primer. ${ }^{16}$ The Ormco primer-Concise porcelain bonding system was significantly stronger than the Scotchprime-Transbond. ${ }^{45}$ In a study published in 2018 , researchers compared All Bond Universal, Adhese Universal, Clearfil Universal Bond, and Single Bond Universal efficiency on etched lithium disilicate glass ceramics after or without silane application, concluding that the non-silanized groups had a reduced bonding effectiveness and additional silane pretreatment helped improve the long-term durability. ${ }^{19}$

Similar shear bond strength values were observed, when testing different bonding materials, on carefully prepared ceramics or enamel.

\section{Feldspathic Ceramic Surface and Ceramic or Composite Brackets}

In general, ceramic brackets show better adhesion to ceramic surfaces, than metallic ones, as shown by. ${ }^{5}$ Hydrofluoric acid also seems a viable solution for bonding, as shown by Harari and his team. ${ }^{46}$ Hydrofluoric acid followed by silane shows better results, ${ }^{47}$ similar to sandblasting and silane, than sandblasting or HF alone. ${ }^{48}$ Similar results, higher SBS than phosphoric acid alone, may be achieved through silica coating and silanization. ${ }^{49}$ Indifferent of type of composite or photopolymerization lamp, the results are consistently within accepted orthodontic range when using HF and silane, but higher for ceramic brackets than metallic ones. ${ }^{50,51}$

Ramos and collaborators found that HF followed by silane reach the best SBS, compared to diamond bur use, orthophosphoric acid $37 \%$ or HF without silane. However, in the case of HF followed by silane treatment of the ceramic, there is a higher risk of damage of the ceramic surface during the debonding stage. ${ }^{52}$

\section{Non-Feldspathic Ceramics}

When talking about newer types of ceramics, there is rarely more than one study researching a certain type of ceramic. 
Table 3 Ceramic or Metallic Brackets Bonded to Different New Ceramic Surfaces in Publishing Order

\begin{tabular}{|c|c|c|c|c|c|}
\hline $\begin{array}{l}\text { No and } \\
\text { Year of } \\
\text { Publication }\end{array}$ & Ceramic Type & $\begin{array}{l}\text { Bracket } \\
\text { Type }\end{array}$ & Adhesive Type & $\begin{array}{l}\text { Additional } \\
\text { Testing }\end{array}$ & Results \\
\hline $1994^{[47]}$ & Feldspathic & Ceramic & Silane/ no silane & & Sgn difference when silane was used \\
\hline $1999^{[40]}$ & $\begin{array}{l}\text { Amalgam/ precious } \\
\text { alloy/ ceramic }\end{array}$ & $\begin{array}{l}\text { Ceramic } \\
\text { Transcend / } \\
\text { Fascination } \\
\text { silanized } \\
\text { brackets }\end{array}$ & $\begin{array}{l}\text { APA/ HF } 9.6 \%+\text { Fuji Ortho LC/ } \\
\text { Photac Bond (GIC) Control } \\
\text { groups used Trandbond or } \\
\text { Concise }\end{array}$ & & $\begin{array}{l}\text { Both GIC satisfactory SBS, silanized } \\
\text { brackets lower SBS, only Fuji on } \\
\text { metal alloy, composites obtained } \\
\text { higher SBS than GIC s }\end{array}$ \\
\hline $2005^{[70]}$ & $\begin{array}{l}\text { Finesse Empress } 2 \\
\text { all ceramic/ } \\
\text { feldspathic Vita } \\
\text { Omega }\end{array}$ & Metallic & $\begin{array}{l}\text { Phosphoric acid + silane+ Unite } \\
\text { Bond }\end{array}$ & & $\begin{array}{l}\text { SBS for Finesse sgn higher, no sgn } \\
\text { difference in ARI scores }\end{array}$ \\
\hline $2006^{[59]}$ & $\begin{array}{l}\text { Feldspathic and } \\
\text { lithium disilicate }\end{array}$ & Metallic & $\begin{array}{l}\text { Silane on glazed/ APA 25um/APA } \\
50 \mathrm{um} / \mathrm{HF} 9.6 \% / 40 \mathrm{um} \text { diamond } \\
\text { bur/ } 60 \mathrm{um} \text { diamond bur }\end{array}$ & Thermocycling & $\begin{array}{l}\text { Lowest SBS HFA sgn difference, } \\
\text { highest SBS diamond burs, lithium } \\
\text { disilicate higher SBS for all groups }\end{array}$ \\
\hline $2006^{[73]}$ & $\begin{array}{l}\text { Low fusing and high } \\
\text { fusing ceramics }\end{array}$ & Metallic & $\begin{array}{l}\text { Resin removal with multifluted } \\
\text { carbide bur with and without } \\
\text { polishing discs }\end{array}$ & & $\begin{array}{l}\text { No sgn difference between the } 2 \\
\text { types of ceramics on debonding } \\
\text { force, no difference between clean- } \\
\text { up methods }\end{array}$ \\
\hline $2007^{[72]}$ & $\begin{array}{l}\text { In-Ceram, IPS } \\
\text { Impress, ceramo- } \\
\text { metal }\end{array}$ & Metallic & & & $\begin{array}{l}\text { Ceramo-metal and In-Ceram } \\
\text { comparable results, IPS_Impress } \\
\text { lower SBS sgn }\end{array}$ \\
\hline $2007^{[58]}$ & $\begin{array}{l}\text { Feldspathic, leucite } \\
\text { based, lithia } \\
\text { disilicate }\end{array}$ & Metallic & $\begin{array}{l}\text { Sandblasting/sandblasting+HF/ } \\
\text { sandblasting+silane /sandblasting } \\
+\mathrm{HF}+\text { silane /tribochemical silica } \\
\text { coating+silane }\end{array}$ & Thermocycling & $\begin{array}{l}\text { Lowest SBS sandblasting only, } \\
\text { feldspathic and lithia disilicate } \\
\text { highest SBS with silica coating, } \\
\text { leucite based HF without silane, } \\
\text { similar to silica coating. }\end{array}$ \\
\hline $2010^{[68]}$ & $\begin{array}{l}\text { IPS Empress } 2 / \text { In- } \\
\text { Ceram alumina }\end{array}$ & $\begin{array}{l}\text { Metallic } \\
\text { and } \\
\text { ceramic }\end{array}$ & $\begin{array}{l}\text { HF } 9.6 \% / \text { phosphoric acid/ } \\
\text { sandblasting for all }\end{array}$ & & $\begin{array}{l}\text { Acid etching sgn increased SBS, } \\
\text { ceramic brackets sgn different } \\
\text { fracture pattern at the adhesive- } \\
\text { bracket interface }\end{array}$ \\
\hline $2010^{[74]}$ & $\begin{array}{l}\text { Aluminous and } \\
\text { fluorapatite ceramic } \\
\text { (Vitadur Alpha/IPS } \\
\text { Emax) }\end{array}$ & $\begin{array}{l}\text { Ceramic } \\
\text { and metallic }\end{array}$ & $\begin{array}{l}\text { Different bracket bases: beads- } \\
\text { Inspire Ice, large round pits } \\
\text { Crystalline IVs, irregular base } \\
\text { Clarity, optimesh stainless } \\
\text { brackets for control }\end{array}$ & & $\begin{array}{l}\text { Highest SBS Inspire Ice sgn different } \\
\text { than the others, all were } \\
\text { satisfactory }\end{array}$ \\
\hline $201 I^{[72]}$ & $\begin{array}{l}\text { Feldspathic, fluoro- } \\
\text { apatite, leucite } \\
\text { reinforced }\end{array}$ & Metallic & $\begin{array}{l}\text { Air particle abrasion } 25 \mathrm{um} / \text { silica } \\
\text { coating } 30 \mathrm{um}\end{array}$ & Thermocycling & $\begin{array}{l}\text { Lowest SBS air particle abrasion } \\
\text { feldspathic and fluoro-apatite, } \\
\text { highest SBS silica coating leucite } \\
\text { reinforced ceramic, silica coating } \\
\text { better overall }\end{array}$ \\
\hline $2014^{[67]}$ & $\begin{array}{l}\text { Monolithic } \\
\text { zirconium oxide } \\
\text { ceramic }\end{array}$ & Metallic & $\begin{array}{l}\text { Glazed/polished +air abrasion } 30 \\
\text { um/air abrasion } 50 \text { um + } \\
\text { Monobond Plus+ Transbond XT } \\
\text { Primer+Transbond XT }\end{array}$ & & Air abrasion improved SBS \\
\hline
\end{tabular}

(Continued) 
Table 3 (Continued).

\begin{tabular}{|c|c|c|c|c|c|}
\hline $2014^{[65]}$ & $\begin{array}{l}\text { Yttria stabilized } \\
\text { tetragonal } \\
\text { polycrystalline } \\
\text { zirconia }\end{array}$ & Metallic & $\begin{array}{l}\text { RelyX adhesive cement and } \\
\text { RelyXUnicemselfadhesive }\end{array}$ & $\begin{array}{l}24 \mathrm{~h} \text { or } 6 \text { months } \\
\text { at } 37 \text { degrees } C\end{array}$ & $\begin{array}{l}\text { Self-adhesive cement RelyXUnicem } \\
\text { not able to sustain bond }\end{array}$ \\
\hline $2016^{[66]}$ & $\begin{array}{l}\text { Feldspathic/ } \\
\text { zirconia }\end{array}$ & Metallic & $\begin{array}{l}\text { Sandblasting all groups }+4 \% \mathrm{HF} \text {, } \\
\text { Porcelain Conditioner silane } \\
\text { Primer/Reliance Assure/ Reliance } \\
\text { Assure Plus/Z Prime Plus }\end{array}$ & & $\begin{array}{l}\text { Tensile bond strength similar for all } \\
\text { groups, silanization after } \\
\text { sandblasting, similar to other } \\
\text { protocols }\end{array}$ \\
\hline $2016^{[56]}$ & $\begin{array}{l}\text { CAD/CAM lithium } \\
\text { disilicate }\end{array}$ & Metallic & $\begin{array}{l}\text { HFA+Silane/ HFA, deglazed/ } \\
\text { glazed }\end{array}$ & & $\begin{array}{l}\text { HF + silane - acceptable SBS values } \\
\text { indifferent of adhesive, but slightly } \\
\text { higher on roughened surface }\end{array}$ \\
\hline $2016^{[69]}$ & $\begin{array}{l}\text { Silica IPS Classic } \\
\text { glazed }\end{array}$ & Metallic & $\begin{array}{l}9.6 \% \mathrm{HF}, 9.6 \% \mathrm{HF}+\text { silane/ } \\
\text { sandblasting+silane/ } \\
\text { tribochemical silica (CoJet) } \\
\text { +silane, all TransbondXT }\end{array}$ & & $\begin{array}{l}\text { HF alone not sufficient SBS, Cojet + } \\
\text { silane and sandblasting+silane } \\
\text { higher than the other groups }\end{array}$ \\
\hline $2017^{[18]}$ & Y-TZP & Metallic & $\begin{array}{l}\text { Abrasion with alumina/ } \\
\text { tribochemical silica coating }+ \\
\text { ESPE-Sil/Alloy Primer/Clearfil/ } \\
\text { Scotchbond }\end{array}$ & +/-thermocycling & $\begin{array}{l}\text { Thermocycling influences results, } \\
\text { better results when mechanically } \\
\text { treated }\end{array}$ \\
\hline $2018^{[55]}$ & $\begin{array}{l}\text { Feldspathic and } \\
\text { lithium disilicate }\end{array}$ & Metallic & $\begin{array}{l}\text { Sandblasting, 9.6\% HF, Transbond } \\
\text { XT/ Assure Plus }\end{array}$ & thermocycling & $\begin{array}{l}\text { Assure Plus better results, } \\
\text { minimized damage on lithium } \\
\text { disilicate }\end{array}$ \\
\hline $2018^{[19]}$ & Lithium disilicate & Ceramic & $\begin{array}{l}\mathrm{HF}+\text { All Bond Universal/Adhese } \\
\text { Universal/ Clearfil Universal } \\
\text { Bond/ Single Bond Universal +/- } \\
\text { silane }\end{array}$ & $\begin{array}{l}\text { Thermocycling, } \\
\text { SEM }\end{array}$ & $\begin{array}{l}\text { Thermocycling influences results. } \\
\text { SBS of the silane groups were } \\
\text { higher and their microleakage } \\
\text { percentages lower than those of } \\
\text { the non-pretreated groups }\end{array}$ \\
\hline $2019^{[51]}$ & IPS e-max CAD & Ceramic & $\begin{array}{l}\mathrm{HF} 60 \mathrm{~s} / \mathrm{S} 3 \mathrm{~min} / \mathrm{HF}+\mathrm{S} / \mathrm{MDP} \\
\text { adhesive followed by resin } \\
\text { cement }\end{array}$ & $\begin{array}{l}\text { SEM, } \\
\text { fractographic } \\
\text { analysis }\end{array}$ & $\begin{array}{l}\text { Monocrystalline brackets with HF } \\
\text { or HF+S chowed highest values, } \\
\text { Polycrystalline with MDP only - } \\
\text { lowest values. }\end{array}$ \\
\hline $2019^{[57]}$ & $\begin{array}{l}\text { IPS E-max and } \\
\text { zirconia }\end{array}$ & Metallic & $\begin{array}{l}\text { E-max HF + MDP primer I coat; } \\
\text { Zirconia MDP I/2/3 coats }\end{array}$ & Thermocycling & $\begin{array}{l}\text { E.max and zirconia with } 3 \text { MDP } \\
\text { primer applications highest SBS }\end{array}$ \\
\hline $2019^{[64]}$ & $\begin{array}{l}\text { Lithium silicate } \\
\text { infused with } \\
\text { zirconia (CELTRA® } \\
\text { DUO, Zirconia and } \\
\text { Lithium disilicate }\end{array}$ & Metallic & $+/-\mathrm{HF}$ & & $\begin{array}{l}\text { SBS of the lithium silicate infused } \\
\text { with zirconia - significantly lower } \\
\text { than HF lithium disilicate group. HF } \\
\text { may increase SBS in Celtra DUO. }\end{array}$ \\
\hline $2020^{[53]}$ & $\begin{array}{l}\text { IPS E-max, CAD/ } \\
\text { CAM, IPS d.Sign } \\
\text { Ceramic fused to } \\
\text { metal }\end{array}$ & Metallic & $\begin{array}{l}\text { Transbond, Light Bond or } \\
\text { Blugloo }+/- \text { HF }\end{array}$ & & $\begin{array}{l}\text { Significant differences in SBS related } \\
\text { to ceramic, surface treatment, and } \\
\text { resin cement. HF etching increased } \\
\text { SBS. }\end{array}$ \\
\hline
\end{tabular}

(Continued) 
Table 3 (Continued).

\begin{tabular}{|l|l|l|l|l|l|}
\hline $2019^{[60]}$ & $\begin{array}{l}\text { Lithium disilicate } \\
\text { and zirconia }\end{array}$ & $\begin{array}{l}\text { Metallic } \\
\text { and } \\
\text { ceramic }\end{array}$ & HF / Phopsphoric acid and silane & $\begin{array}{l}\text { HF use on zirconia and lithium- } \\
\text { disilicate, does not cause a } \\
\text { significant increase in SBS, } \\
\text { compared to etching with PhA and } \\
\text { silane application. HF can weaken } \\
\text { the surface structure. }\end{array}$ \\
\hline $2019^{[21]}$ & Zirconia & Ceramic & $\begin{array}{l}\text { MDP ceramic primer + } \\
\text { orthodontic primer / universal } \\
\text { adhesive / MDP ceramic primer } \\
+ \text { universal adhesive. }\end{array}$ & Thermocycling & $\begin{array}{l}\text { For ceramic brackets to zirconia, } \\
\text { ceramic primer used with an } \\
\text { orthodontic primer, rather than } \\
\text { using a universal adhesive, is } \\
\text { recommended. }\end{array}$ \\
\hline $2020^{[22]}$ & $\begin{array}{l}\text { Lithium disilicate E- } \\
\text { max and lithium } \\
\text { silicate infused with } \\
\text { zirconia (CELTRA } \\
\text { DUO) }\end{array}$ & Ceramic & $\begin{array}{l}\text { aluminium oxide air abrasion + 3 } \\
\text { different brackets }\end{array}$ & $\begin{array}{l}\text { Mean SBS of the E-max groups } \\
\text { were significantly less than } \\
\text { CELTRA } \mathbb{D} \text { DUO. Only the Symetri } \\
\text { bracket was effective for both } \\
\text { substrates. }\end{array}$ \\
\hline
\end{tabular}

On etchable ceramics, hydrofluoric acid increases shear bond strength. ${ }^{53}$

On lithium disilicate ceramics, IPS ceramic etching gel $^{\mathrm{TM}}$ and Monobond plus achieved better results than the one step system, containing etching and primer. ${ }^{54,55}$ Feldspathic and lithium disilicate were bonded with either Transbond primer or Assure Plus after sandblasting and etching with 9.6\% HF. Bracket bond to lithium disilicate by Assure Plus was significantly stronger than that to feldspathic porcelain. Cohesive porcelain fracture had the lowest frequency in the lithium disilicate/Assure Plus group. When working on CAD/CAM lithium disilicate, hydrofluoric acid followed by silane shows acceptable SBS values indifferent of adhesive, but slightly higher on roughened surface ${ }^{56}$ or MDP adhesive. ${ }^{57}$ On feldspathic, leucite based and lithium disilicate samples, testing showed lowest SBS when sandblasting only, feldspathic and lithium disilicate highest SBS with silica coating, leucite based ceramics showed highest SBS values for HF without silane, similar to silica coating. ${ }^{58}$ Contradicting these findings, only one study shows that on both feldspathic and lithium disilicate ceramics, lowest SBS was achieved with HF, at a statistically significant difference and highest SBS was achieved when using diamond burs. Lithium disilicate showed higher SBS for all groups. ${ }^{59}$ Another study compared HF use to phosphoric acid and silane on both dilithium silicate and zirconia, showing no significant advantage of HF use. However,
SBS in zirconia groups was lower than in lithium disilicate for both procedures. ${ }^{60}$

On Vita Enamic CAD/CAM ceramics, CoJet coating gives better results than HF acid which are also higher than diamond bur roughening and phosphoric acid use. ${ }^{61}$

When testing different surface preparations on feldspathic, leucite, leucite free, fluorapatite ceramics, researchers found no difference between ceramic types in terms of SBS, just that leucite and leucite-free ceramics differed most with respect to their surface roughness. Conditioning with conventional 5\% HF or sandblasting resulted in significantly higher bond strengths, than with $9.6 \% \mathrm{HF}^{62}$

When comparing Empress 2, Finesse, Ceramco II, Barceló et $\mathrm{al}^{63}$ found that deglazing helps adhesion for all types.

On non-etchable, zirconia-based ceramics, different types of surface preparation methods have been investigated. Mechanical preparation of the surfaces through particle abrasion or polishing with silicon carbide paper achieved better results than untreated surfaces on Yttria stabilized tetragonal polycrystalline zirconia ceramic surfaces. ${ }^{18}$ Three coats of MDP primer showed better results than one coat on zirconia ceramics. ${ }^{57}$ When compared to universal adhesive or orthodontic adhesive, MDP showed better results ${ }^{21}$ On lithium silicate infused with zirconia, Celtra DUO, SBS was significantly lower than the hydrofluoric acid pre-treated lithium disilicate group. Acid etching may, however, improve adhesion to 
this new type on ceramics. ${ }^{64}$ When using aluminium oxide air abrasion, mean SBS of an E-max CAD group were significantly less than the CELTRA ${ }^{\circledR}$ DUO group, showing efficacity of this method on the new ceramic material. ${ }^{22}$ On Yttria stabilized tetragonal polycrystalline zirconia, RelyX self-adhesive cement was not able to sustain the bond, no matter if new generation primers such as MDP and tribochemical silica were used. ${ }^{65}$ When comparing sandblasted feldspathic to zirconia ceramics and different primers, such as Assure, Assure plus, Porcelain Conditioner or $\mathrm{Z}$ prime, the researchers found similar tensile bond strengths on all surfaces. ${ }^{66}$ Air abrasion improved SBS on monolithic zirconium oxide ceramic when using Monobond and Transbond. ${ }^{67}$

Abu Alhaija and collaborators tested IPS Empress II and In-Ceram alumina. They sandblasted and acid treated surfaces with either HF $9.6 \%$ or phosphoric acid, bonded metallic and ceramic brackets and reached the following conclusions: acid etching significantly increased SBS, the fracture pattern at the adhesivebracket interface is significantly different for ceramic brackets, compared to metallic brackets and no significant difference was found between the two ceramic types. ${ }^{68}$ Some tests on IPS silica ceramics revealed that hydrofluoric acid alone does not achieve satisfactory SBS and the combination of CoJet and silane or sandblasting and silane give higher values than the other groups. ${ }^{69}$ By comparing IPS Empress II and Finesse all ceramic to feldspathic ceramic, the first two achieved significantly higher SBS when phosphoric acid and Unite were used. ${ }^{70}$

When testing either feldspathic, fluoro-apatite, leucite reinforced ceramics, researchers found the lowest SBS on air particle abraded feldspathic and fluoro-apatite and highest SBS on silica coated leucite reinforced ceramic, silica coating showed better overall results. ${ }^{71}$

Ceramo-metal and In-Ceram showed comparable results, IPS Impress had significantly lower SBS values. ${ }^{72}$

On low fusing and high fusing ceramics, no statistically significant difference was found between the two types of ceramics on debonding force and no difference between clean-up methods. $^{73}$

On aluminous and fluorapatite ceramic, Vitadur Alpha and IPS Emax, researchers tested different bracket base designs, either beads, large round pits, irregular base and one group of stainless steel brackets mesh base. Beads in Inspire Ice brackets showed the highest SBS, significantly different than the other groups. The SBS of all base designs could withstand normal orthodontic force. ${ }^{74}$

\section{Photopolymerization Light Sources}

Gonçalves compared different etching times, 20s or $60 \mathrm{~s}$ when $10 \%$ HF was used, together with 4 different light sources: halogen, led, argon laser or plasma arc. Researchers found no difference between light sources and statistically significant lower shear bond strength for 20 s etching. ${ }^{75}$ When comparing halogen curing unit for $10 \mathrm{~s}$ or $20 \mathrm{~s}$ to a LED unit for 3.5 or $10 \mathrm{~s}$ in standard or fast mode, Elekdag-Turk and collaborators found no statistically significant differences between SBS, ARI scores, porcelain damage and ceramic bracket base fracture. ${ }^{76}$

In opposition to this, De Abreu Neto shows that thermocycling and type of photopolymerization lamp influences shear bond strength. ${ }^{15}$ Türkkahraman also finds significantly higher SBS for LED lamp than halogen when bonding ceramic brackets to porcelain surfaces in thermocycled samples, ${ }^{77}$ which is also supported by another study. ${ }^{78}$ When comparing VALO Ortho Cordless appliance with irradiance $3200 \mathrm{~mW} / \mathrm{cm} 2$ for $3 \mathrm{~s}$ and Optilight Max appliance with irradiance $1200 \mathrm{~mW} / \mathrm{cm} 2$ for $40 \mathrm{~s}$, researchers found no significant difference in SBS. ${ }^{79}$

\section{Conclusion}

Some types of adhesive may achieve minimal bond strength (6-8 MPa) even on glazed ceramic. Ceramic surface preparation may be done by sandblasting or HF acid with generally similar results. When using hydrofluoric acid, 60s application and $9.6 \%$ solution are advised. Studies rarely show any statistical difference and there are reduced number of samples in most studies. Ceramic brackets show better adhesion to ceramic surfaces, than metallic ones and the same bonding protocol may be used. A higher bond strength may lead to ceramic surface damage of the prosthetic restoration.

Concerning non feldspathic, newer types of ceramics, there is rarely more than one study researching a certain type of ceramic. Therefore, additional research is necessary. General rules seem to apply to these categories also.

There is no clear evidence that a certain type of photopolymerization device produces higher SBS values.

\section{Abbreviations}

HF, hydrofluoric acid; APA, air particle abrasion; SEM, scanning electron-microscopy; SBS, shear bond strength; 
CSBS, cyclic shear bond strength; Sgn, statistically significant; TS, tribochemical silica coating.

\section{Acknowledgments}

The authors wish to thank the "Iuliu Hatieganu' Medicine and Pharmacy University for providing the opportunity to do interesting research.

\section{Disclosure}

The authors declare that there is no conflict of interest regarding the publication of this paper.

\section{References}

1. Edward A, McLaren DS, Tran CP. DDS ceramics in dentistry-part i: classes of materials. inside dentistry. Available from: http://www. insidedentistry.net. Accessed March 2, 2021.

2. Jorquera G, Merino N, Walls S, Mahn E, Fernández E. Simplified classification for dental ceramics. J Dent Sci Ther. 2016;1(2):22-25.

3. Giordano R, McLaren EA. Ceramics overview: classification by microstructure and processing methods. Compend Contin Educ Dent. 2010;31(9):682-4, 686, 688 passim; quiz 698, 700.

4. Arango Santander S, Vargas AP, Escobar JS, Monteiro FJ. Ceramics for dental restorations - an introduction. Dyna Rev Fac Nac Minas. 2010;77:26-36.

5. Shenoy A, Shenoy N. Dental ceramics: an update. J Conserv Dent. 2010;13(4):195-203. doi:10.4103/0972-0707.73379

6. Goldstein RE, Parkins FM. Air-abrasive technology: its new role in restorative dentistry. $J$ Am Dent Assoc. 1994;125(5):551-557. doi:10.14219/jada.archive.1994.0077

7. Abu Alhaija ES, Al-Wahadni AM. Evaluation of shear bond strength with different enamel pre-treatments. Eur J Orthod. 2004;26 (2):179-184. doi:10.1093/ejo/26.2.179

8. Reynolds IR. A review of direct orthodontic bonding. $\mathrm{Br} J$ Orthod. 1975;2(3):171-178. doi:10.1080/0301228X.1975.11743666

9. Joseph VP, Rossouw E. The shear bond strengths of stainless steel and ceramic brackets used with chemically and light-activated composite resins. Am J Orthod Dentofacial Orthop. 1990;97(2):121-125. doi:10.1016/0889-5406(90)70084-P

10. Forsberg CM, Hagberg C. Shear bond strength of ceramic brackets with chemical or mechanical retention. Br J Orthod. 1992;19 (3):183-189. doi:10.1179/bjo.19.3.183

11. Franklin S, Garcia-Godoy F. Shear bond strengths and effects on enamel of two ceramic brackets. J Clin Orthod. 1993;27(2):83-88.

12. Viazis AD, Cavanaugh G, Bevis RR. Bond strength of ceramic brackets under shear stress: an in vitro report. Am J Orthod Dentofacial Orthop. 1990;98(3):214-221. doi:10.1016/S0889-5406(05)81598-7

13. Finnema KJ, Ozcan M, Post WJ, Ren Y, Dijkstra PU. In-vitro orthodontic bond strength testing: a systematic review and meta-analysis. Am J Orthod Dentofacial Orthop. 2010;137(5):615-622.e3. doi:10.1016/j.ajodo.2009.12.021

14. Bourke BM, Rock WP. Factors affecting the shear bond strength of orthodontic brackets to porcelain. Br J Orthod. 1999;26(4):285-290. doi:10.1093/ortho/26.4.285

15. De Abreu Neto HF, Costa AR, Correr AB, et al. Influence of light source, thermocycling and silane on the shear bond strength of metallic brackets to ceramic. Braz Dent J. 2015;26(6):685-688 doi:10.1590/0103-6440201300416
16. Matos NR, Costa AR, Valdrighi HC, et al. Effect of acid etching, silane and thermal cycling on the bond strength of metallic brackets to ceramic. Braz Dent J. 2016;27(6):734-738. doi:10.1590/01036440201601077

17. Huang TH, Kao CT. The shear bond strength of composite brackets on porcelain teeth. Eur J Orthod. 2001;23(4):433-439. doi:10.1093/ ejo/23.4.433

18. Kim NH, Kim YJ, Lee DY. Bond strengths of orthodontic metal brackets to tribochemically silica-coated zirconia surfaces using different 10-methacryloyloxydecyl dihydrogen phosphate-containing primers. Int J Adhesion Adhesives. 2017;74(1):21-29. doi:10.1016/j. ijadhadh.2016.12.006

19. Yao C, Yang H, Yu J, Zhang L, Zhu Y, Huang C. High bond durability of universal adhesives on glass ceramics facilitated by silane pretreatment. Oper Dent. 2018;43(6):602-612. doi:10.2341/17-227-L

20. Aguiar AP, Costa AR, Bortolazzo Correr A, et al. Effect of hydrofluoric acid concentration and thermal cycling on the bond strength of brackets to ceramic. Braz Dent J. 2019;30(6):587-591. doi:10.1590/ 0103-6440201902989

21. Ju GY, Oh S, Lim BS, Lee HS, Chung SH. Effect of simplified bonding on shear bond strength between ceramic brackets and dental zirconia. Materials. 2019;12(10):1640. doi:10.3390/ma12101640

22. Oldham CC, Ballard RW, Yu Q, Kee EL, Xu X, Armbruster PC. In vitro comparison of shear bond strengths of ceramic orthodontic brackets with ceramic crowns using an aluminium oxide air abrasion etchant. Int Orthod. 2020;18(1):115-120. doi:10.1016/j. ortho.2019.07.005

23. Pannes DD, Bailey DK, Thompson JY, Pietz DM. Orthodontic bonding to porcelain: a comparison of bonding systems. J Prosthet Dent. 2003;89(1):66-69. doi:10.1067/mpr.2003.63

24. White GJ, Tyas MJ. The bond strength of orthodontic resins to porcelain. Aust Orthod J. 1993;13(1):8-12.

25. Barbosa VL, Almeida MA, Chevitarese O, Keith O. Direct bonding to porcelain. Am J Orthod Dentofacial Orthop. 1995;107(2):159-164. doi:10.1016/S0889-5406(95)70131-1

26. Lifshitz AB, Cárdenas M. A comparison between the shear bond strength of brackets bonded to glazed and deglazed porcelain surfaces with resin-reinforced glass-ionomer cement and a bis-GMA resin adhesive. World J Orthod. 2006;7(2):134-137.

27. Nebbe B, Stein E. Orthodontic brackets bonded to glazed and deglazed porcelain surfaces. Am J Orthod Dentofacial Orthop. 1996;109(4):431-436. doi:10.1016/S0889-5406(96)70125-7

28. Zachrisson YO, Zachrisson BU, Büyükyilmaz T. Surface preparation for orthodontic bonding to porcelain. Am $J$ Orthod Dentofacial Orthop. 1996;109(4):420-430. doi:10.1016/S0889-5406(96)70124-5

29. Cochrane D, O'Keefe KL, Turner DT, Powers JM. Bond strength of orthodontic composite cement to treated porcelain. Am J Orthod Dentofacial Orthop. 1997;111(3):297-300. doi:10.1016/S08895406(97)70188-4

30. Chung CH, Brendlinger EJ, Brendlinger DL, Bernal V, Mante FK. Shear bond strengths of two resin-modified glass ionomer cements to porcelain. Am J Orthod Dentofacial Orthop. 1999;115(5):533-535. doi:10.1016/S0889-5406(99)70275-1

31. Bishara SE, Ajlouni R, Oonsombat C, Laffoon J. Bonding orthodontic brackets to porcelain using different adhesives/enamel conditioners: a comparative study. World J Orthod. 2005;6(1):17-24.

32. Trakyali G, Malkondu O, Kazazoğlu E, Arun T. Effects of different silanes and acid concentrations on bond strength of brackets to porcelain surfaces. Eur J Orthod. 2009;31(4):402-406. doi:10.1093/ ejo/cjn118

33. Larmour CJ, Bateman G, Stirrups DR. An investigation into the bonding of orthodontic attachments to porcelain. Eur J Orthod. 2006;28(1):74-77. doi:10.1093/ejo/cji072 
34. Stella JP, Oliveira AB, Nojima LI, Marquezan M. Four chemical methods of porcelain conditioning and their influence over bond strength and surface integrity. Dental Press J Orthod. 2015;20 (4):51-56. doi:10.1590/2176-9451.20.4.051-056.oar

35. Gillis I, Redlich M. The effect of different porcelain conditioning techniques on shear bond strength of stainless steel brackets. Am J Orthod Dentofacial Orthop. 1998;114(4):387-392. doi:10.1016/ S0889-5406(98)70183-0

36. Alzainal AH, Majud AS, Al-Ani AM, Mageet AO. Orthodontic bonding: review of the literature. Int J Dent. 2020;2020:8874909. doi:10.1155/2020/8874909

37. Ajlouni R, Bishara SE, Oonsombat C, Soliman M, Laffoon J. The effect of porcelain surface conditioning on bonding orthodontic brackets. Angle Orthod. 2005;75(5):858-864. doi:10.1043/0003$3219(2005) 75$

38. Schmage P, Nergiz I, Herrmann W, Ozcan M. Influence of various surface-conditioning methods on the bond strength of metal brackets to ceramic surfaces. Am J Orthod Dentofacial Orthop. 2003;123 (5):540-546. doi:10.1016/S0889-5406(02)56911-0

39. Sant'Anna EF, Monnerat ME, Chevitarese O, Stuani MB. Bonding brackets to porcelain-in vitro study. Braz Dent J. 2002;13 (3):191-196. doi:10.1590/S0103-64402002000300010

40. Jost-Brinkmann PG, Böhme A. Shear bond strengths attained in vitro with light-cured glass ionomers vs composite adhesives in bonding ceramic brackets to metal or porcelain. Adhes Dent. 1999;1 (3):243-253.

41. Costa AR, Correr AB, Puppin-Rontani RM, et al. Effect of bonding material, etching time and silane on the bond strength of metallic orthodontic brackets to ceramic. Braz Dent J. 2012;23(3):223-227. doi:10.1590/S0103-64402012000300007

42. Costa AR, Correr AB, Consani S, et al. Influence of water storage and bonding material on bond strength of metallic brackets to ceramic. Braz Dent J. 2015;26(5):503-506. doi:10.1590/01036440201300403

43. Sundareswaran S, Usman U. Orthodontic possibilities on ceramic surfaces with 10-methacryloyloxydecyle dihydrogen phosphate. Orthodontics (Chic). 2012;13(1):e29-e36.

44. Abdelnaby YL. Effects of cyclic loading on the bond strength of metal orthodontic brackets bonded to a porcelain surface using different conditioning protocols. Angle Orthod. 2011;81(6):1064-1069. doi:10.2319/030211-151.1

45. Zelos L, Bevis RR, Keenan KM. Evaluation of the ceramic/ceramic interface. Am J Orthod Dentofacial Orthop. 1994;106(1):10-21. doi:10.1016/S0889-5406(94)70016-8

46. Harari D, Shapira-Davis S, Gillis I, Roman I, Redlich M. Tensile bond strength of ceramic brackets bonded to porcelain facets. $\mathrm{Am}$ J Orthod Dentofacial Orthop. 2003;123(5):551-554. doi:10.1016/ S0889-5406(02)56913-4

47. Whitlock BO, Eick JD, Ackerman RJ, Glaros AG, Chappell RP. Shear strength of ceramic brackets bonded to porcelain. $\mathrm{Am}$ J Orthod Dentofacial Orthop. 1994;106(4):358-364. doi:10.1016/ S0889-5406(94)70056-7

48. Kocadereli I, Canay S, Akça K. Tensile bond strength of ceramic orthodontic brackets bonded to porcelain surfaces. Am J Orthod Dentofacial Orthop. 2001;119(6):617-620. doi:10.1067/ mod.2001.113655

49. Ozcan M, Vallittu PK, Peltomäki T, Huysmans MC, Kalk W. Bonding polycarbonate brackets to ceramic: effects of substrate treatment on bond strength. Am J Orthod Dentofacial Orthop. 2004;126 (2):220-227. doi:10.1016/j.ajodo.2003.06.015

50. Al-Hity R, Gustin MP, Bridel N, Morgon L, Grosgogeat B. In vitro orthodontic bracket bonding to porcelain. Eur J Orthod. 2012;34 (4):505-511. doi:10.1093/ejo/cjr043

51. Di Guida LA, Benetti P, Corazza PH, Della Bona A. The critical bond strength of orthodontic brackets bonded to dental glass-ceramics. Clin Oral Investig. 2019;23(12):4345-4353. doi:10.1007/s00784-019-02881-5
52. Ramos TF, Lenza MA, Reges RR, Freitas G. Influence of ceramic surface treatment on shear bond strength of ceramic brackets. Indian J Dent Res. 2012;23(6):789-794. doi:10.4103/0970-9290.111261

53. Juntavee P, Kumchai H, Juntavee N, Nathanson D. Effect of ceramic surface treatment and adhesive systems on bond strength of metallic brackets. Int J Dent. 2020;2020:7286528. doi:10.1155/ 2020/7286528

54. Asiry MA, AlShahrani I, Alaqeel SM, Durgesh BH, Ramakrishnaiah R. Effect of two-step and one-step surface conditioning of glass ceramic on adhesion strength of orthodontic bracket and effect of thermo-cycling on adhesion strength. J Mech Behav Biomed Mater. 2018;84:22-27. doi:10.1016/j.jmbbm.2018.04.021

55. Naseh R, Afshari M, Shafiei F, Rahnamoon N. Shear bond strength of metal brackets to ceramic surfaces using a universal bonding resin. J Clin Exp Dent. 2018;10(8):e739-e745. doi:10.4317/jced.54175

56. Lestrade AM, Ballard RW, Xu X, Yu Q, Kee EL, Armbruster PC. Porcelain surface conditioning protocols and shear bond strength of orthodontic brackets. Aust Orthod J. 2016;32(1):18-22.

57. da Silva Milagres F, Oliveira DD, Silveira GS, Oliveira EF, da Gama Antunes AN. Bond strength and failure pattern of orthodontic tubes adhered to a zirconia surface submitted to different modes of application of a ceramic primer. Materials. 2019;12(23):3922. doi:10.3390/ ma12233922

58. Karan S, Büyükyilmaz T, Toroğlu MS. Orthodontic bonding to several ceramic surfaces: are there acceptable alternatives to conventional methods? Am J Orthod Dentofacial Orthop. 2007;132(2):144. e7-14. doi:10.1016/j.ajodo.2006.12.006

59. Türk T, Saraç D, Saraç YS, Elekdağ-Türk S. Effects of surface conditioning on bond strength of metal brackets to all-ceramic surfaces. Eur J Orthod. 2006;28(5):450-456. doi:10.1093/ejo/cj1010

60. Mehmeti B, Kelmendi J, Iiljazi-Shahiqi D, et al. Comparison of shear bond strength orthodontic brackets bonded to zirconia and lithium disilicate crowns. Acta Stomatol Croat. 2019;53(1):17-27. doi:10.15644/asc53/1/2

61. Elsaka SE. Influence of surface treatments on bond strength of metal and ceramic brackets to a novel CAD/CAM hybrid ceramic material. Odontology. 2016;104(1):68-76. doi:10.1007/s10266-014-0188-8

62. Falkensammer F, Freudenthaler J, Pseiner B, Bantleon HP. Influence of surface conditioning on ceramic microstructure and bracket adhesion. Eur J Orthod. 2012;34(4):498-504. doi:10.1093/ejo/ cjr034

63. Barceló Santana HF, Hernández Medina R, Acosta Torres SL, Sánchez Herrera LM, Fernández Pedrero AJ, Ortíz González R. Evaluation of bond strength of metal brackets by a resin to ceramic surfaces. J Clin Dent. 2006;17(1):5-9.

64. Gardiner R, Ballard R, Yu Q, Kee E, Xu X, Armbruster P. Shear bond strength of orthodontic brackets bonded to a new all-ceramic crown composed of lithium silicate infused with zirconia: an in vitro comparative study. Int Orthod. 2019;17(4):726-732. doi:10.1016/j.ortho.2019.08.011

65. da Silva EM, Miragaya L, Sabrosa CE, Maia LC. Stability of the bond between two resin cements and an yttria-stabilized zirconia ceramic after six months of aging in water. $J$ Prosthet Dent. 2014;112(3):568-575. doi:10.1016/j.prosdent.2013.12.003

66. Mehta AS, Evans CA, Viana G, Bedran-Russo A, Galang-Boquiren MT. Bonding of metal orthodontic attachments to sandblasted porcelain and zirconia surfaces. Biomed Res Int. 2016;2016:5762785. doi: $10.1155 / 2016 / 5762785$

67. Bavbek NC, Roulet JF, Ozcan M. Evaluation of microshear bond strength of orthodontic resin cement to monolithic zirconium oxide as a function of surface conditioning method. $J$ Adhes Dent. 2014;16 (5):473-480. doi:10.3290/j.jad.a32812

68. Abu Alhaija ES, Abu AlReesh IA, AlWahadni AM. Factors affecting the shear bond strength of metal and ceramic brackets bonded to different ceramic surfaces. Eur J Orthod. 2010;32(3):274-280. doi:10.1093/ejo/cjp098 
69. Zhang ZC, Qian YF, Yang YM, Feng QP, Shen G. Bond strength of metal brackets bonded to a silica-based ceramic with light-cured adhesive: influence of various surface treatment methods. J Orofac Orthop. 2016;77(5):366-372. doi:10.1007/s00056-016-0044-2

70. Chay SH, Wattanapayungkul P, Yap AU, Loh PL, Chung SM. Comparison of the bond strength of stainless steel orthodontic brackets bonded to crown porcelains. Aust Orthod J. 2005;21(1):19-23.

71. Saraç YŞ, Külünk T, Elekdağ-Türk S, Saraç D, Türk T. Effects of surface-conditioning methods on shear bond strength of brackets bonded to different all-ceramic materials. Eur J Orthod. 2011;33 (6):667-672. doi:10.1093/ejo/cjq132

72. Abu Alhaija ES, Al-Wahadni AM. Shear bond strength of orthodontic brackets bonded to different ceramic surfaces. Eur J Orthod. 2007;29 (4):386-389. doi:10.1093/ejo/cjm032

73. Ferri NP, Eliades T, Zinelis S, Bradley TG. Force to debond brackets from high-fusing and low-fusing porcelain systems. Angle Orthod. 2006;76(2):278-281

74. Kukiattrakoon B, Samruajbenjakul B. Shear bond strength of ceramic brackets with various base designs bonded to aluminous and fluorapatite ceramics. Eur J Orthod. 2010;32(1):87-93. doi:10.1093/ejo/ cjp055
75. Gonçalves PR, Moraes RR, Costa AR, et al. Effect of etching time and light source on the bond strength of metallic brackets to ceramic. Braz Dent J. 2011;22(3):245-248. doi:10.1590/S010364402011000300011

76. Elekdag-Turk S, Sarac YS, Turk T, Sarac D. The effect of a light-emitting diode on shear bond strength of ceramic brackets bonded to feldspathic porcelain with different curing times. Eur J Orthod. 2007;29(3):299-303. doi:10.1093/ejo/cjm017

77. Türkkahraman H, Küçükesmen HC. Porcelain surface-conditioning techniques and the shear bond strength of ceramic brackets. Eur J Orthod. 2006;28(5):440-443. doi:10.1093/ejo/cj1026

78. Türkkahraman H, Küçükeşmen HC. Effects of light-emitting diode and halogen light curing techniques on ceramic brackets bonded to porcelain surfaces. Angle Orthod. 2006;76(4):673-676. doi:10.1043/ 0003-3219(2006)076

79. Vallandro Lopes G, Correr-Sobrinho L, Bortolazzo Correr A, et al. Light activation and thermocycling methods on the shear bond strength of brackets bonded to porcelain surfaces. Braz Dent J. 2020;31(1):52-56. doi:10.1590/0103-6440202003101

\section{Publish your work in this journal}

Clinical, Cosmetic and Investigational Dentistry is an international, peer-reviewed, open access, online journal focusing on the latest clinical and experimental research in dentistry with specific emphasis on cosmetic interventions. Innovative developments in dental materials, techniques and devices that improve outcomes and patient satisfaction and preference will be highlighted. The manuscript management system is completely online and includes a very quick and fair peer-review system, which is all easy to use. Visit http://www.dovepress.com/testimonials.php to read real quotes from published authors. 